\title{
A FINITE ELEMENT MODEL FOR A HIGHER-ORDER SHEAR-DEFORMABLE BEAM THEORY
}

\author{
T. Kant And A. Gupta \\ Department of Civil Engineering, Indian Institute of Technology, \\ Bombay 400 076, India
}

(Received 19 March 1987, and in revised form 12 November 1987)

\begin{abstract}
The theory for a higher order shear-deformable beam model is first developed. It is based on a higher order displacement model and incorporates linear and quadratic variation of transverse normal strain and transverse shearing strain respectively through the beam thickness. The effects of the transverse normal and shear stresses are included in the definition of the material's constitutive law. The warping of the transverse normal crosssection of the beam is automatically incorporated in the mathematical model. The question of selecting a shear correction coefficient as in a first-order shear deformable Timoshenko theory does not arise. A linear two-noded finite element model of this theory is introduced and developed next. Both static and free vibration results of this theory are presented and compared with those of Euler and Timoshenko theories for various boundary and loading conditions.
\end{abstract}

\section{INTRODUCTION}

Structural behaviour of beams may be satisfactorily approximated by the elementary Euler-Bernoulli theory of bending. The main assumption in this theory is that transverse normals to the reference middle plane remain so during bending, implying transverse shear strain becomes zero. Thus bending rotation becomes a first derivative of the transverse displacement $w$ and hence requires the transverse displacement field $C^{1}$ continuous. Both compatible and incompatible and complicated higher order $C^{1}$ continuous elements have been derived in the past $[1,2]$.

The Euler theory may lead to serious discrepancies in cases of deep beams with small aspect ratios $(L / h)$ where shear effects are not insignificant. Timoshenko [3] extended the domain of validity of this theory by incorporating the effect of transverse shear, constant through the thickness, into the governing equations. This extension involves a shear coefficient which is somewhat arbitrary. Cowper [4] and recently Murty [5, 6], have analyzed expressions for this coefficient. For rectangular cross-sections, it is taken to be $5 / 6$ as have taken in the formulations to follow here. The discrepancies, however, are large in the case of built-up beams even after refining the values of shear coefficient.

In recognition of the need for a more refined theoretical model, a simple higher order theory is developed here to include all secondary effects: i.e., the transverse shear stress and strain, its parabolic variation across the thickness, and the normal direct transverse stress and strain due to the change in thickness. A similar effort has also been made by Reddy [7], but his model results in $C^{1}$ continuity and does not take into account transverse normal strain.

In the finite element discretization, our theoretical model requires use of the simple $C^{0}$ continuous shape functions and thus the development of the resulting computer code becomes an easy task. 


\section{THEORY}

The theory is based on the Taylor series expansion of the displacement components in the following form:

$$
\begin{gathered}
U(x, z)=u(x)+z \theta(x)+z^{2} u^{*}(x)+z^{3} \theta^{*}(x)+\cdots, \\
W(x, z)=w(x)+z \psi(x)+z^{2} w^{*}(x)+z^{3} \psi^{*}(x)+\cdots,
\end{gathered}
$$

Here $x$ and $z$ are the axial and the transverse co-ordinate directions, the $x$ axis being assumed to lie at the mid-depth of the beam. The parameters $U$ and $W$ are the displacement components at a general point $(x, z)$ in the $x$ and $z$ directions respectively. The parameters $u, u^{*}, \theta, \theta^{*}, w, w^{*}$, and $\psi, \psi^{*}$, are the corresponding terms in the Taylor series expansion and are all defined along the $x$ axis at $z=0$.

In the case of pure bending of a beam with no axial deformation of the reference $x$ axis, the parameters $u$ and $u^{*}$ will vanish. Further, in order to match the powers of $z$ in the expressions for the transverse shear strain due to $U$ and $W$, it is clearly seen that the parameters $\psi$ and $\psi^{*}$ should vanish. Thus the displacement forms appropriate to the theory developed here for pure flexure of beams will reduce to,

$$
U(x, z)=z \theta(x)+z^{3} \theta^{*}(x), \quad W(x, z)=w(x)+z^{2} w^{*}(x)
$$

The expansions (2a) and (2b) contain the minimum number of terms to predict the improved bending modes of deformation of the beam and include the effects of transverse shear deformation with warping of the transverse cross-section and transverse normal strain. Further, the transverse shear strain due to axial displacement component $U$ is of the same order in $z$ as those determined by the transverse displacement component $W$. In expressions (2a) and (2b) the term $\theta$ is the rotation of the normal to the reference axis in the $x-z$ plane and the terms $w$ define the transverse displacement of the reference $x$-axis. While these terms are physical quantities, the parameters $\theta^{*}$ and $w^{*}$ are the higher order terms in the Taylor series expansion and their interpretation in terms of physical quantities is difficult. Thus the generalized displacement $\mathbf{\delta}$ of the reference plane is expressed in terms of four independent variables as

$$
\boldsymbol{\delta}=\left(w, \theta, w^{*}, \theta^{*}\right)^{t} .
$$

The strain components are then related to the components of the generalized displacement vector $\delta$ by the relations

$$
\begin{gathered}
\varepsilon_{x}=\partial U / \partial x=z \chi+z^{3} \chi^{*}, \\
\gamma_{x z}=\partial U / \partial z+\partial W / \partial x=\phi+z^{2} \phi^{*}, \quad \varepsilon_{z}=z\left(2 w^{*}\right) .
\end{gathered}
$$

The curvature terms $\chi, \chi^{*}$ and shearing strains $\phi, \phi^{*}$ along with $2 w^{*}$ form the generalized strain vector $\bar{\varepsilon}$ and they are related to the generalized displacement vector $\delta$ by

$$
\overline{\boldsymbol{\varepsilon}}=\left[\begin{array}{c}
\chi \\
\phi \\
\chi^{*} \\
\phi^{*} \\
2 w^{*}
\end{array}\right]=\left[\begin{array}{cccc}
0 & \mathrm{~d} / \mathrm{d} x & 0 & 0 \\
\mathrm{~d} / \mathrm{d} x & 1 & 0 & 0 \\
0 & 0 & 0 & \mathrm{~d} / \mathrm{d} x \\
0 & 0 & \mathrm{~d} / \mathrm{d} x & 3 \\
0 & 0 & 2 & 0
\end{array}\right]\left[\begin{array}{c}
w \\
\theta \\
w^{*} \\
\theta^{*}
\end{array}\right]=\underline{L} .
$$

The total potential energy $\pi$ is given by,

$$
\pi=\frac{1}{2} \int_{l} \overline{\boldsymbol{\varepsilon}}^{\mathrm{t}} \overline{\mathbf{\sigma}} \mathrm{d} x-\int_{l}\left(p_{z}^{+}+p_{z}^{-}\right)\left(w+\frac{h^{2}}{4} w^{*}\right) \mathrm{d} x,
$$


where $p_{z}^{+}$and $p_{z}^{-}$are the transverse distributed loads on the positive and negative extreme $z$ planes respectively and $h$ is the total thickness of the beam. The generalized stress vector $\overline{\boldsymbol{\sigma}}$ which is the integral of the physical stress components through the cross-section is given by

$$
\overline{\boldsymbol{\sigma}}=\left(M, Q, M^{*}, Q^{*}, M_{z}^{*}\right)^{\mathrm{t}}
$$

Here,

$$
\begin{gathered}
Q=\int_{A} \tau_{x z} \mathrm{~d} A, \quad\left[M, M_{z}^{*}\right]=\int_{A}\left[\sigma_{x}, \sigma_{z}\right] z \mathrm{~d} A, \\
Q^{*}=\int_{A} \tau_{x z} z^{2} \mathrm{~d} A, \quad M^{*}=\int_{A} \sigma_{x} z^{3} \mathrm{~d} A .
\end{gathered}
$$

Thus the generalized stress-strain relationship is

$$
\overline{\mathbf{\sigma}}=\left[\begin{array}{c}
M \\
Q \\
M^{*} \\
Q^{*} \\
M_{z}^{*}
\end{array}\right]=\left[\begin{array}{ccccc}
C_{11} I & 0 & C_{11} I^{*} & 0 & C_{13} I \\
0 & C_{66} A & 0 & C_{66} I & 0 \\
C_{11} I^{*} & 0 & C_{11} I^{* *} & 0 & C_{13} I^{*} \\
0 & C_{66} I & 0 & C_{66} I^{*} & 0 \\
C_{13} I & 0 & C_{13} I^{*} & 0 & C_{33} I
\end{array}\right]\left[\begin{array}{c}
\chi \\
\phi \\
\chi^{*} \\
\phi^{*} \\
2 w^{*}
\end{array}\right]=D \bar{\varepsilon}
$$

Here

$$
\begin{gathered}
I=\int_{A} z^{2} \mathrm{~d} A, \quad I^{*}=\int_{A} z^{4} \mathrm{~d} A, \quad I^{* *}=\int_{A} z^{6} \mathrm{~d} A, \\
C_{11}=C_{33}=E /\left(1-\nu^{2}\right), \quad C_{13}=\nu E /\left(1-\nu^{2}\right), \quad C_{66}=G .
\end{gathered}
$$

\section{A FINITE ELEMENT MODEL}

The energy expression (6) contains only the first derivatives of the components of the generalized displacement vector $\delta$ and thus only $C^{0}$ continuity is required for the shape function to be used in the element formulation. If the same shape function is used to define all the components of the generalized displacement vector $\delta$ then

$$
\boldsymbol{\delta}=\sum_{i=1}^{n} N_{i}(x) \boldsymbol{\delta}_{i}
$$

where $N_{i}$ is the shape function associated with node $i, \boldsymbol{\delta}_{i}$ is the value of $\boldsymbol{\delta}$ corresponding to node $i$ and $n$ is the number of nodes in the element. In the present formulation,

$$
n=2, \quad N_{1}(x)=1-x / l, \quad N_{1}(x)=x / l,
$$

$l$ being the length of the element.

With the aid of expressions (5) and (9), $\bar{\varepsilon}$ at any point can be expressed in terms of $\boldsymbol{\delta}_{i}$ as follows:

$$
\overline{\boldsymbol{\varepsilon}}=\underline{L} \boldsymbol{\delta}=\underline{L} \sum_{i=1}^{n} N_{i}(x) \delta_{i}=\sum_{i=1}^{n} \underline{B}_{i} \boldsymbol{\delta}_{i}, \quad \underline{B}_{i}=\underline{L} N_{i}(x) .
$$

After having obtained the $D$ and $B$ matrices from expressions (8) and (11), the element stiffness matrix $\underline{K}^{e}$ and mass matrix $\underline{M}^{e}$ can be computed as follows:

$$
\underline{K}^{e}=\int_{1} \underline{B}^{\mathrm{t}} \underline{D} \underline{B} \mathrm{~d} x
$$




$$
\underline{M}^{e}=\int_{I} \underline{N}^{t} \rho\left[\begin{array}{cccc}
A & 0 & 0 & 0 \\
0 & I & 0 & 0 \\
0 & 0 & I^{*} & 0 \\
0 & 0 & 0 & I^{* *}
\end{array}\right] \underline{N} \mathrm{~d}
$$

The consistent load vector $\mathbf{P}$ due to distributed load $p_{z}^{+}$can be written in general as

$$
\begin{aligned}
\mathbf{P} & =\int_{A} \underline{N}^{\mathrm{t}} p_{z}^{+} \mathrm{d} A \\
& =\left(\frac{p_{z}^{+} l}{2}, 0, \frac{p_{z}^{+} l h^{2}}{8}, 0, \frac{p_{z}^{+} l}{2}, 0, \frac{p_{z}^{+} l h^{2}}{8}, 0\right)^{\mathrm{t}} .
\end{aligned}
$$

A FORTRAN computer code has been developed based on the foregoing approach to generate the element mass and stiffness matrices and then the solution $[8,9]$ of the system of equations for static and eigenvalue problems.

\section{NUMERICAL EXAMPLE}

Both static deflection and free-vibration analyses have been carried out by using the present formulation. These results are compared with those of a similar finite element formulation of a Timoshenko beam [8] and also with those of the finite element formulation of Euler beam.

The static deflections for a cantilever beam with a tip load are shown in Figure 1, and those with a uniformly distributed load in Figure 2. The eigenvalues of this beam are shown in Figures 7-9.

Similar results are presented for simply supported and fixed beams under a concentrated load at the centre and a uniformly distributed load. They are given in Figures 3-6, and 10-15 respectively.

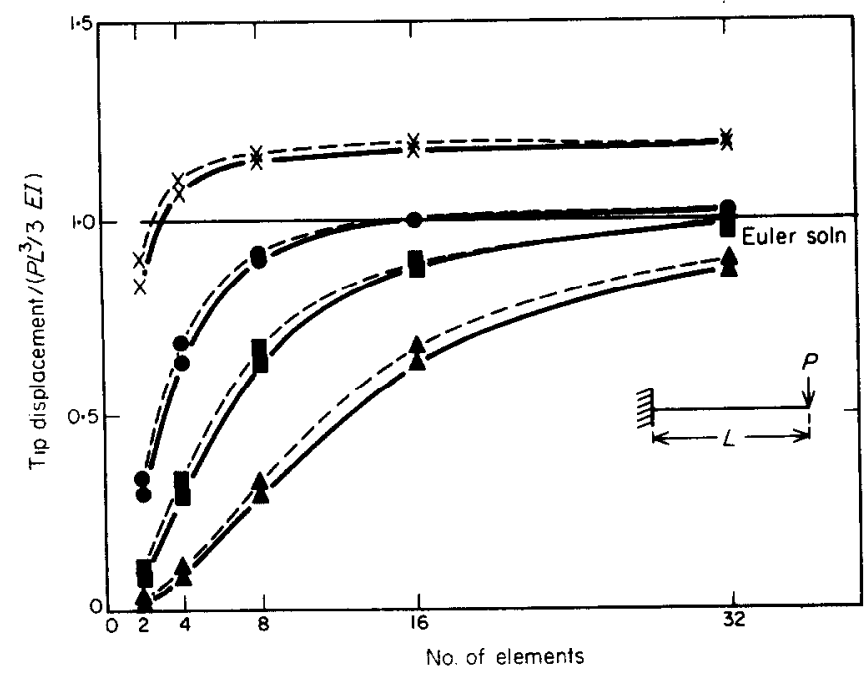

Figure 1. Cantilever under tip load. - - , Timoshenko; - higher order. $\times, L / h=2 \cdot 0 ; 0, L / h=5 \cdot 0$; $L / h=10 \cdot 0 ; \Delta, L / h=20 \cdot 0$. 


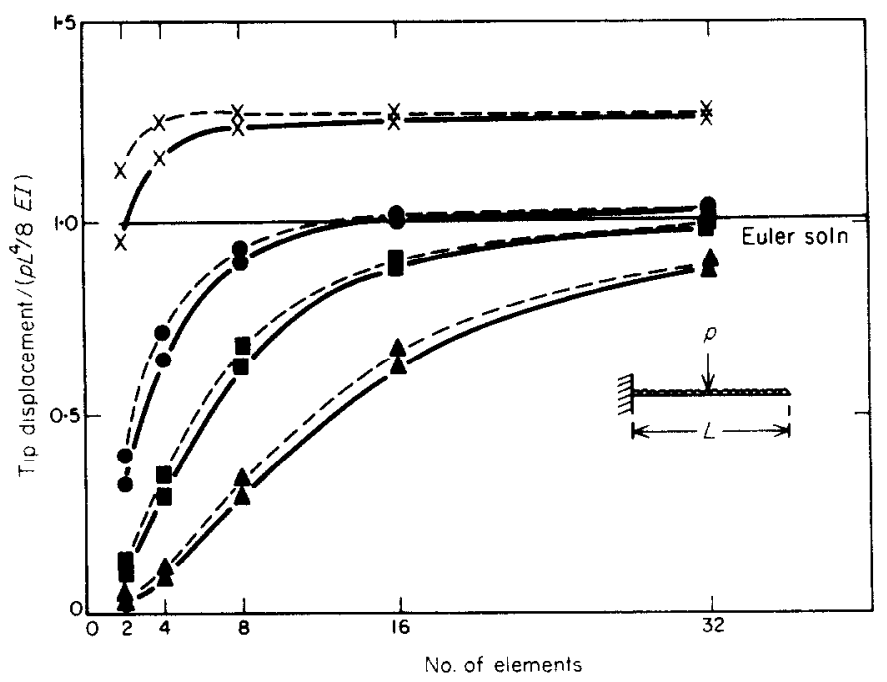

Figure 2. Cantilever under uniformly distributed load. Key as Figure 1.

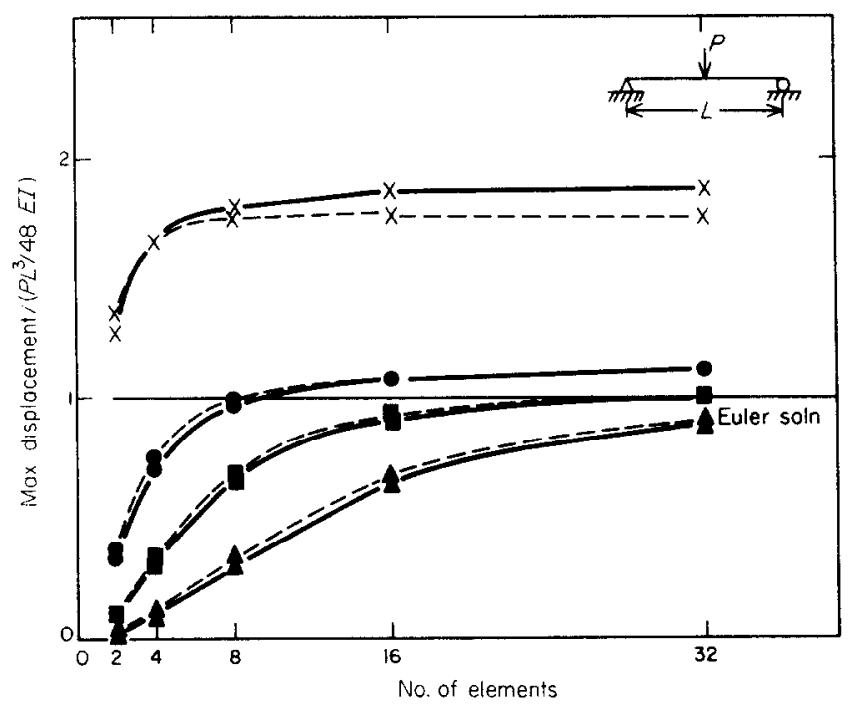

Figure 3. Simply supported beam under central load. Key as Figure 1.

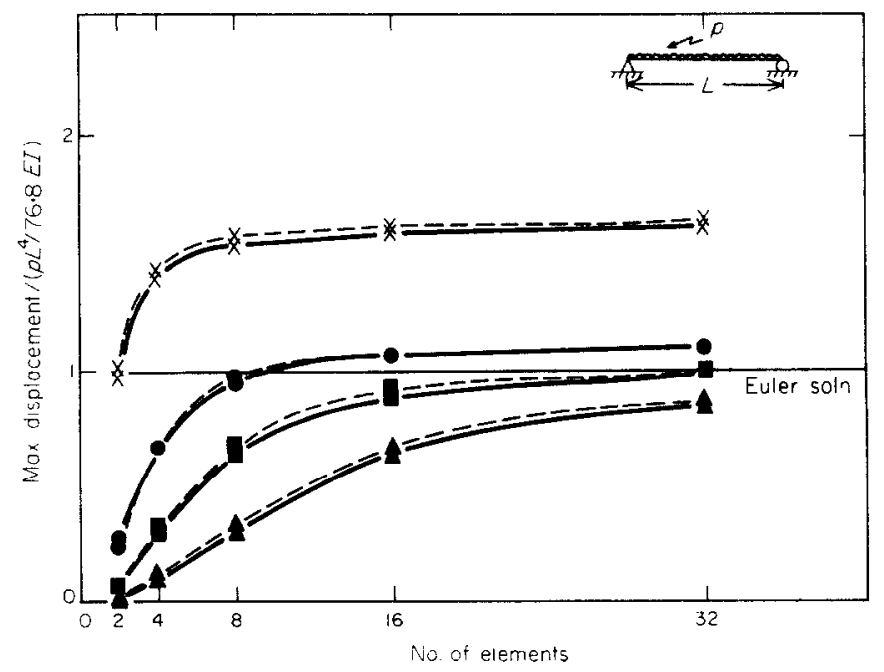

Figure 4. Simply supported beam under uniformly distributed load. Key as Figure 


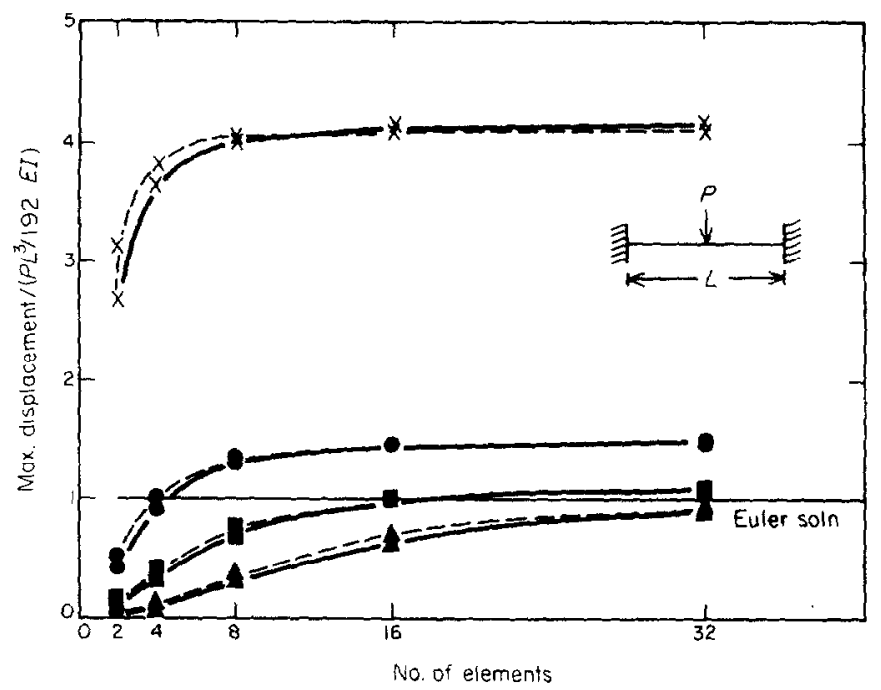

Figure 5. Fixed beam under central point load, Key as Figure 1.

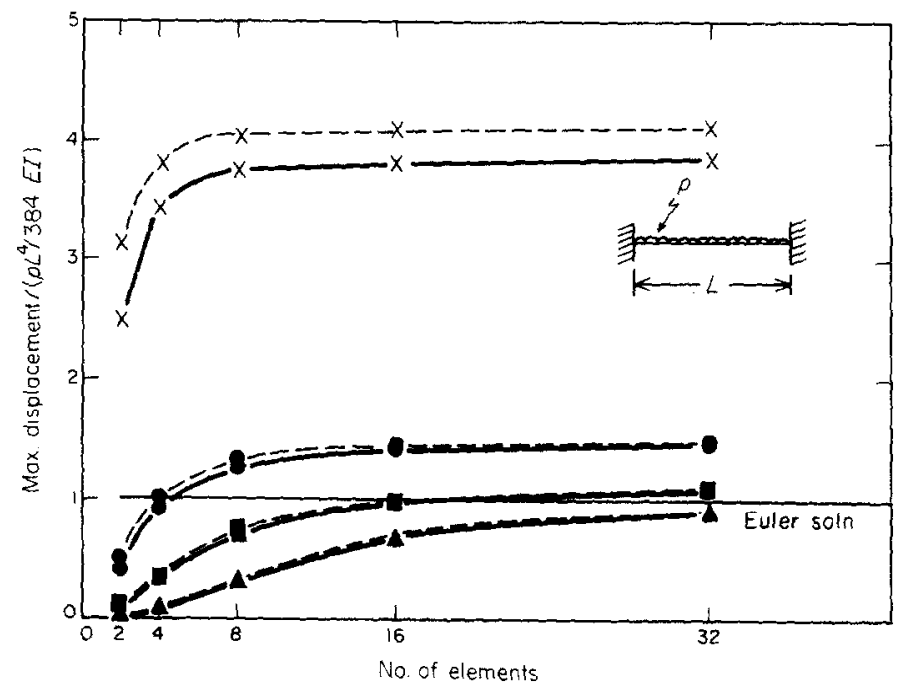

Figure 6. Fixed beam under uniformly distributed load. Key as Figure 1.

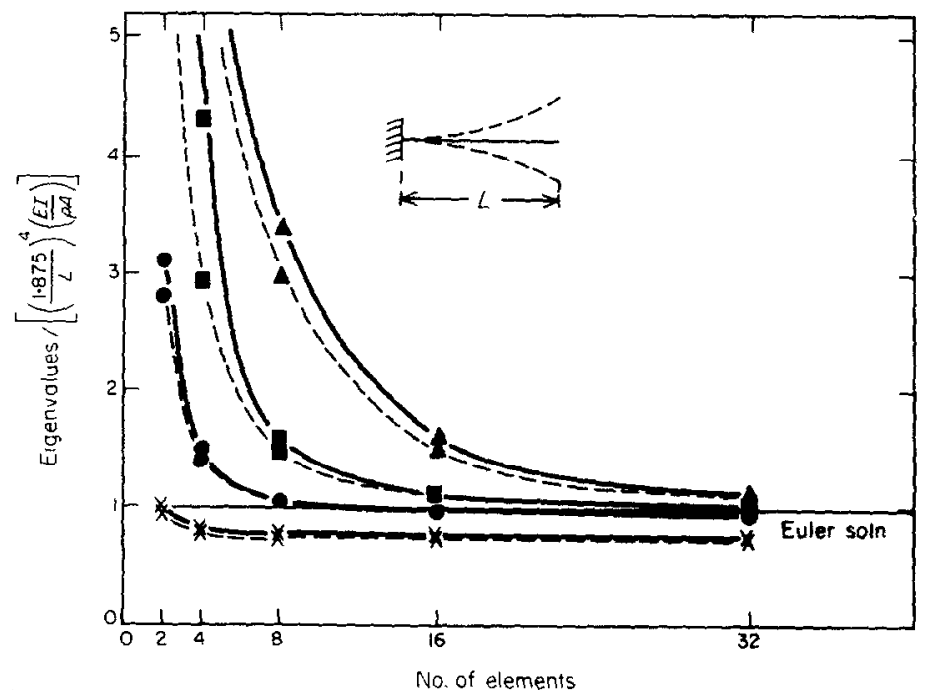

Figure 7. Cantilever beam in first mode of vibration. Key as Figure 1. Eigenvalues $=\omega^{2}=(\text { angular frequency })^{2}$ 


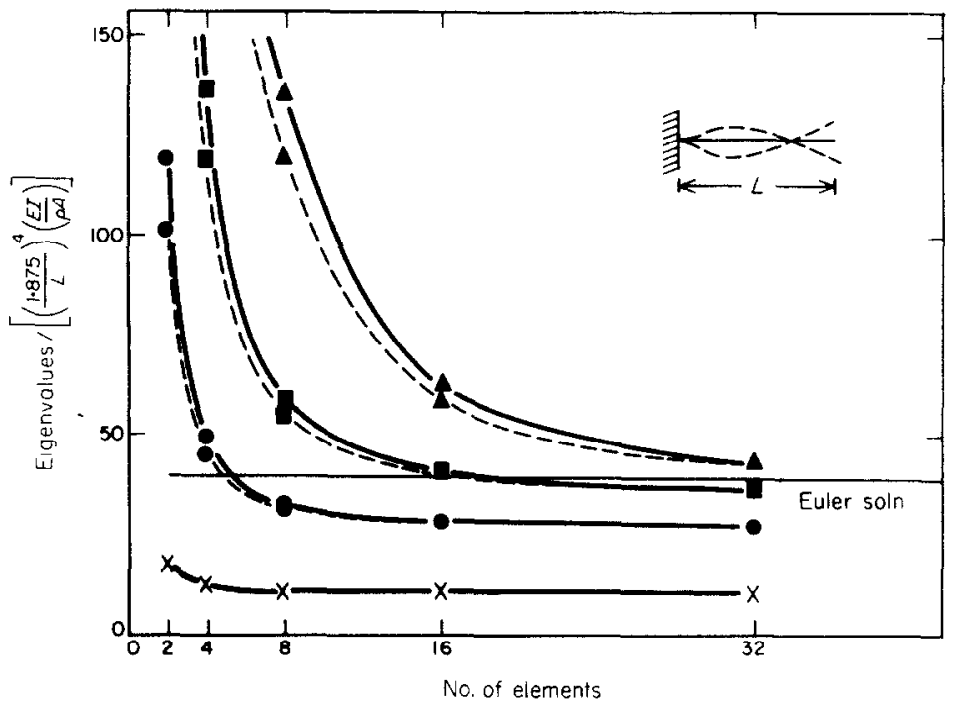

Figure 8. Cantilever beam in second mode of vibration. Key as Figure 7.

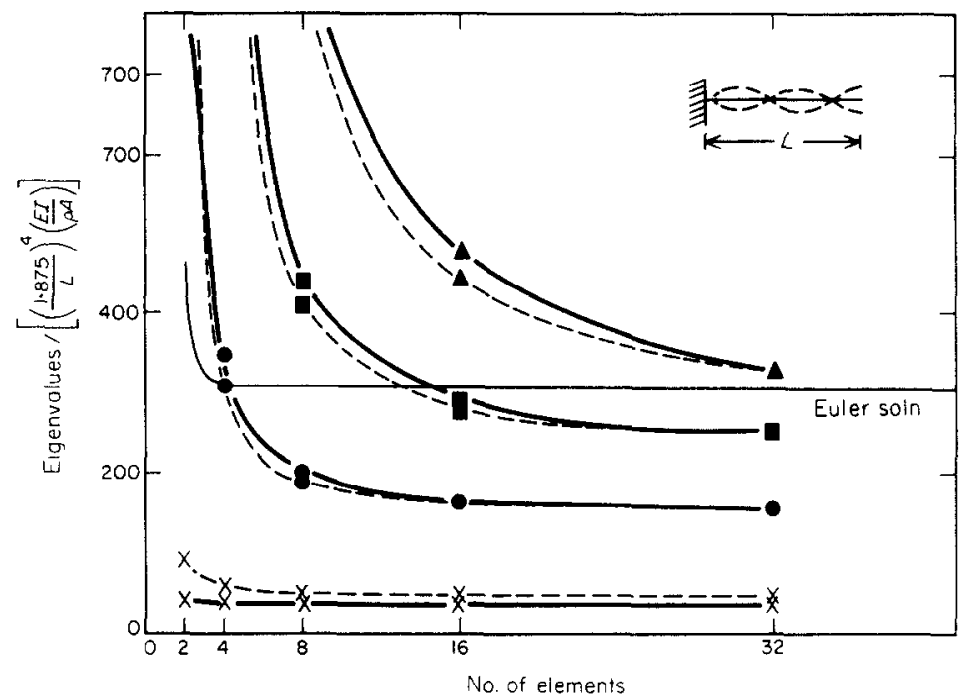

Figure 9. Cantilever beam in third mode of vibration. Key as Figure 7.

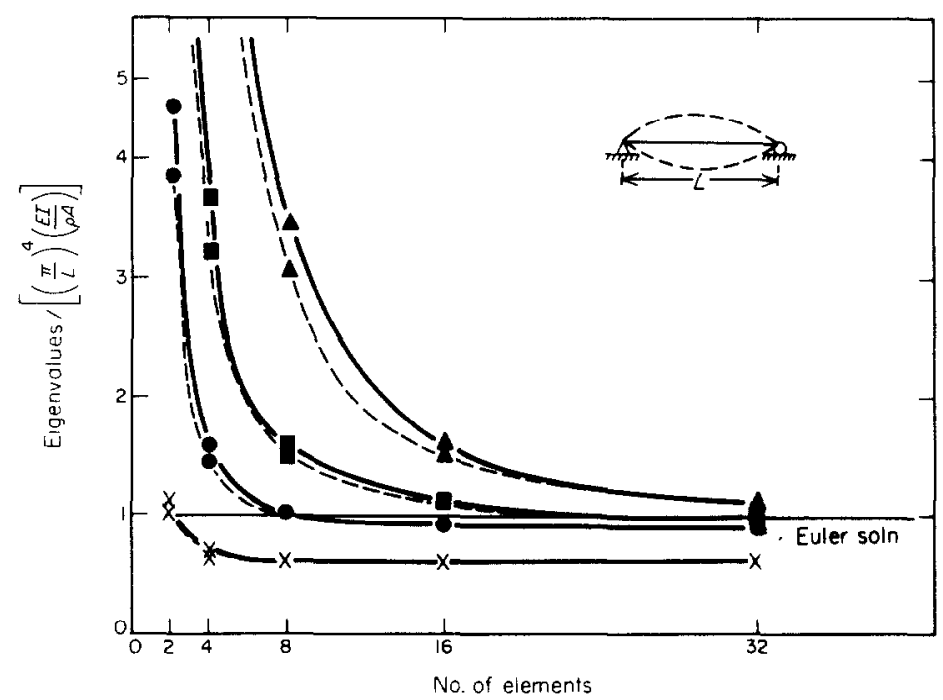

Figure 10. Simply supported beam in first mode of vibration. Key as Figure 7. 


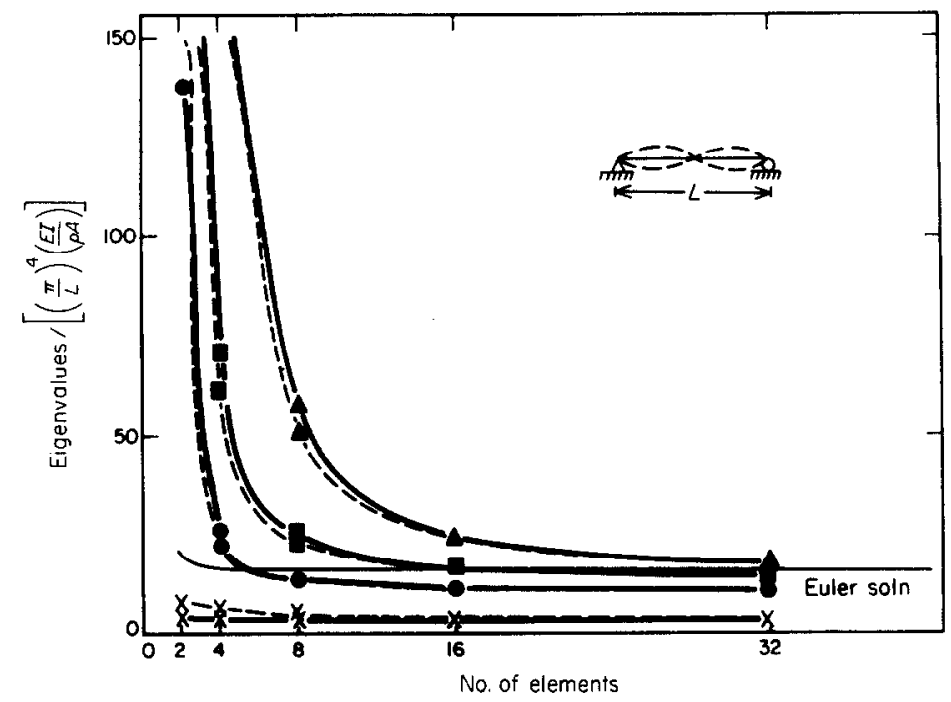

Figure 11. Simply supported beam in second mode of vibration. Key as Figure 7

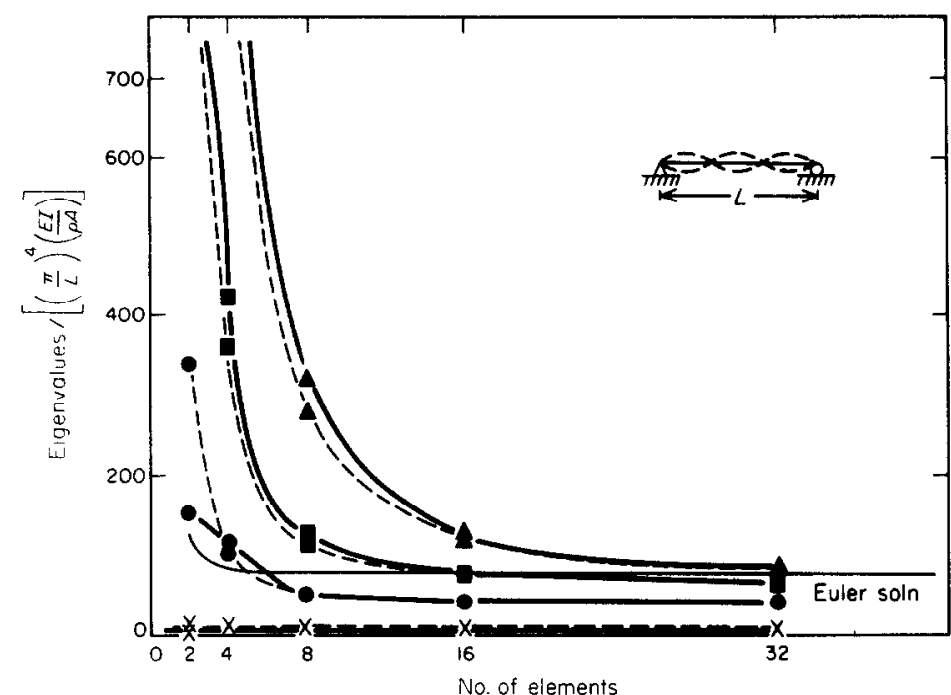

Figure 12. Simply supported beam in third mode of vibration. Key as Figure 7.

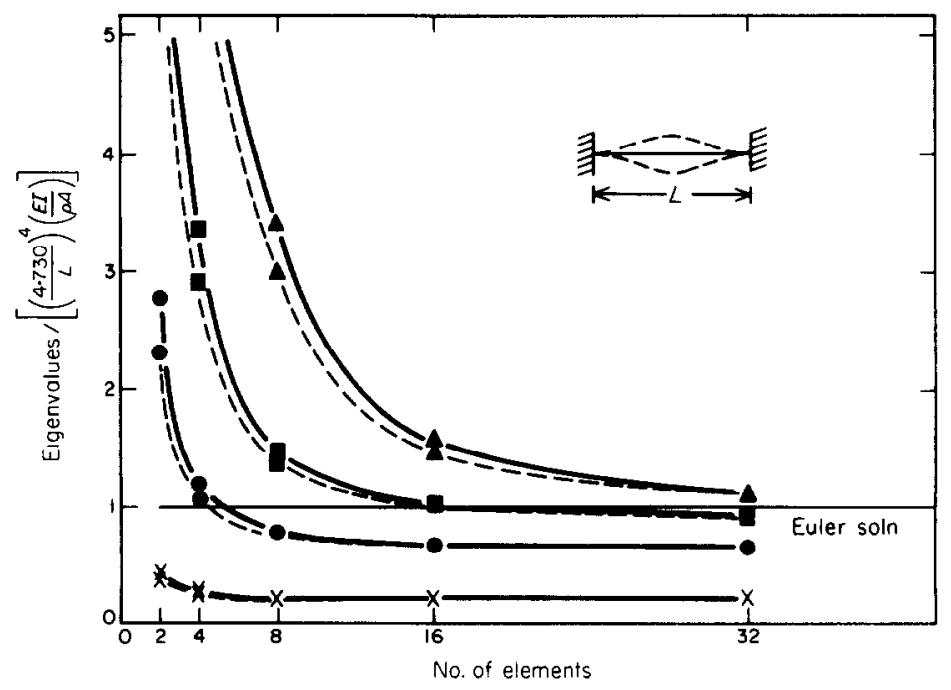

Figure 13. Fixed beam in first mode of vibration. Key as Figure 7. 


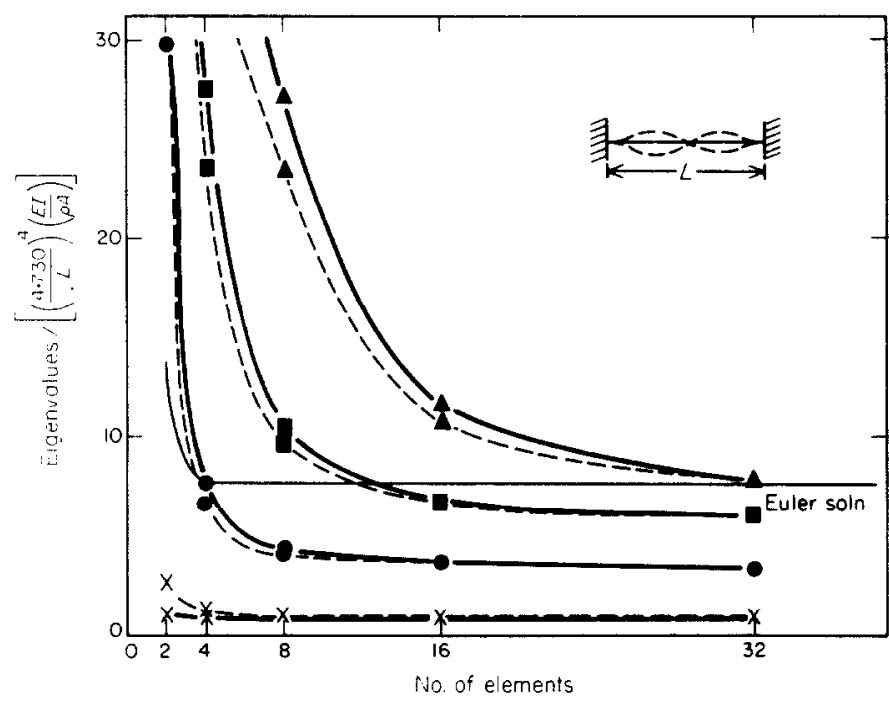

Figure 14. Fixed beam in second mode of vibration. Key as Figure 7.

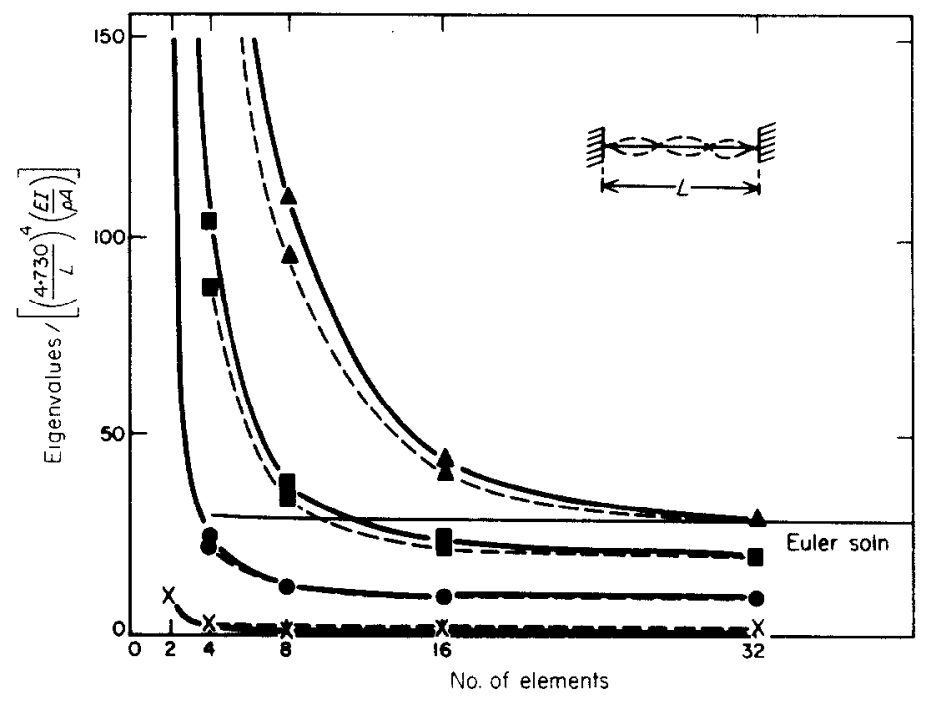

Figure 15. Fixed beam in third mode of vibration. Key as Figure 7.

\section{CONCLUSIONS}

It is well known that the effects of transverse shear deformation and normal stress in the thickness direction become important above a certain value of the thickness-length parameter. The present formulation incorporates a quadratic variation of transverse shearing strain and linear variation of transverse normal strain through the thickness of beam. Results for both the displacements and eigenvalues are in excellent agreement with those of Timoshenko theory. From the results it can be seen that for thick beams the classical theory underpredicts displacements and overpredicts natural frequencies owing to the neglect of transverse shear energy. For thin beams the convergence, though slower, seems to be to the results which will again confirm the above statement. The deviation from classical theory would not be much in this case. 
For moderately thick beams, the present higher order formulation predicts displacements a little smaller than does Timoshenko theory while in the case of frequencies the case is the reverse. The deviation from Timoshenko theory in vibration problems increases in the higher modes of vibration. In the case of very thick beams, say with $L / h=2$ the results sometimes do not converge in the expected way. This happens because the thick beam no longer behaves like a flexural beam.

The theory could be useful in analyses of non-homogeneous, anisotropic, composite or sandwich systems, as the mathematical model on which this theory is based is far superior to the earlier ones. The formulation described here offers a convenient and concise method for the analysis of both thick and thin beams. Further, it is believed that the results presented here will form a basis for future comparative studies.

\section{REFERENCES}

1. G. P. BAZeley, Y. K. Cheung, B. M. Irons and O. C. ZienkiewiCZ 1965 Proceedings 1st Conference on Matrix Methods in Structural Mechanics, AFFDL-TR-60-80, 547-576. Triangular elements in bending, conforming and nonconforming solutions.

2. K. BELL and I. HOLLAND 1969 Report, Technical University of Norway, Tapir. Finite Element methods in stress analysis.

3. S. P. Timoshenko 1921 Philosphical Magazine, Series 6, 41, 744-746. On the correction for shear of differential equation for transverse vibration of prismatic bars.

4. G. R. COWPER, 1966 American Society of Mechanical Engineers Journal of Applied Mechanics, 33, 335-340. The shear coefficient in Timoshenko beam theory.

5. A. V. K. MURTY 1970 American Institute of Aeronautics and Astronautics Journal, 8, 34-38. Vibration of short beams.

6. A. V. K. MuRTy 1970 American Institute of Astronautics and Astronomics Journal, 8, 11, 2098-2100. Analysis of short beams.

7. J. N. REDDY 1984 Energy and Variational Methods in Applied Mechanics, New York: John Wiley.

8. E. HINTON and D. R. J. OWEN 1979 An Introduction to Finite Element Computations, Swansea, U.K.: Pineridge Press.

9. K. J. BATHE and E. L. WILSON 1976 Numerical Methods in Finite Element Analysis. Englewood Cliffs, N.J.: Prentice-Hall. 